\title{
Profiling of Atmospheric Water Vapor from the SSM/T-2 Radiometric Measurements
}

\author{
J. R. Wang \\ Laboratory for Hydrospheric Processes \\ NASA Goddard Space Flight Center, Greenbelt, Maryland 20771 \\ tel: 301-286-8949, fax: 301-286-1762, email: wang@sensor.gsfc.nasa.gov
}

\section{ABSTRACT}

An advantage of using the millimeter-wave measurements for water vapor profiling is the ability to probe beyond a moderate cloud cover. Wang et al [1] demonstrated such a capability from an airborne MIR (Millimeter-wave Imaging Radiometer) flight over the Pacific Ocean during an intense observation period of TOGA/COARE (Tropical Ocean Global Atmosphere/ Couple Ocean Atmospheric Response Experiment) in early 1993. A Cloud Lidar System (CLS) [2] and MODIS Airborne Simulator (MAS) [3] were on board the same aircraft to identify the presence of clouds and cloud type. The retrieval algorithm not only provides output of a water vapor profile, but also the cloud liquid water and approximate cloud altitude required to satisfy convergence of the retrieval. The validity of these cloud parameters has not been verified previously. In the following, these cloud parameters are compared with those derived from concurrent measurements from the CLS and AMPR (Advanced Microwave Precipitation Radiometer).

\section{THE MEASUREMENT}

The measurements were made by the MIR, CLS, MAS, and AMPR on borad the NASA ER-2 aircraft on 17-18 January 1993. The aircraft was stationed in Townsville $\left(\sim 19.4^{\circ} \mathrm{S}\right.$ and $146.8^{\circ} \mathrm{E}$ ), Australia. The plane took off around 2300 UTC on January 17 , climbed to cruising altitude of about $19 \mathrm{~km}$, and headed in a nearly straight path toward a region of convection centered at about $1.5^{\circ} \mathrm{S}$ and $156^{\circ} \mathrm{E}$. Fig. 1 gives variations of signals from the CLS (top portion) and two selected channels of the MAS between 2331-0035 UTC during the transit. The CLS backscatter signals displays the locations of the clouds as well as surface return at $0 \mathrm{~km}$; data gaps at $0 \mathrm{~km}$ indicates total attenuation of CLS surface return signals by the overlying clouds. The two MAS channelsa at 0.875 and $10.4 \mu \mathrm{m}$ gives radiances from the clouds. There are two distinct types of clouds; the first one is the high cirrus clouds that occur mainly above $8 \mathrm{~km}$, and the second one is the low liquid clouds that occur at altitudes $\leq 5 \mathrm{~km}$. The low-level liquid clouds strongly attenuate the CLS surface return signals. Both channels from the MAS respond to cirrus and liquid clouds. While the 0.875 $\mu \mathrm{m}$ channel appears to be affected more strongly by the liquid clouds, the $10.4 \mu \mathrm{m}$ channel shows a higher sensitivity to the cirrus clouds. It was shown [1] that the millimeter-wave measuements at frequencies $\leq 220 \mathrm{GHz}$ respond mainly to the liquid clouds. The presence of cirrus clouds at altitudes $\geq 8 \mathrm{~km}$ has a negligible effect on the MIR measurements.

\section{RETRIEVALS OF WATER VAPOR PROFILES}

The algorithm (a physical iterative process) used for retrieving water vapor profiles from the millimeter-wave radiometric measurements has been reported elsewhere $[1$, 4] and therefore will not be repeated here. During TOGA/COARE the MIR measurements were made at the six frequency channels of $89,150,183.3 \pm 1,183.3 \pm 3$, $183.3 \pm 7$, and $220 \mathrm{GHz}$ [5]. Results of the retrievals from the MIR data, acquired over the same period as that in Fig. 1 , are shown in Fig. 2. Plot (A) of the figure gives the profiles of water vapor mixing ratio displayed in gray scale. Plots (B) and (C), for 6-channel and 5-channel (excluding the $220 \mathrm{GHz}$ ) retrievals respectively, show the amount of cloud liquid water (CLW) and the height of the cloud top required by the algorithm to obtain a convergent retrieval [1]. All except a few cases of the retrievals during this entire period are convergent. Plot (A) shows that there is an enhanced water vapor in the region of liquid clouds $(\sim 0026$ UTC) and that the cirrus clouds have negligible impact on water vapor profiling. Retrievals using 5-channel and 6channel data give small differences in the retrieved water vapor profiles. However, there are some subtle differences between plots (B) and $(\mathrm{C})$ due to the selection of the altitude levels ( 5 levels for 5 -channel retrievals) where water vapor and cloud parameters are estimated. There are differences in both the estimated heights of the cloud tops and CLW's. There is a limited validation of the retrieved water vapor mixing ratio [4]. The CLW values required to bring about convergent retrievals have not been quantitatively verified, although the variations of CLW and cloud tops in plots (B) and (C) of Figure 2 appear to correspond well with those observed in Fig. 1.

\section{CLOUD LIQUID WATER ESTIMATION FROM AMPR}

The AMPR measurements were made at 10.7, 19.35, 37 and $85 \mathrm{GHz}[6]$. When the brightness temperatures $\left(\mathrm{T}_{b}\right)$ at these channels are plotted along the same time period as in Fig. 1, a close association is found between the variations of $\mathrm{T}_{\mathrm{b}}$ values and CLS and MAS signals from liquid clouds. There are some minor problems with the AMPR operation during TOGA/COARE, and not all channels are functioning properly at all times. As a result, the measurements may not be of sufficient accuracy for use in the estimation of CLW. To minimize the errors caused by these instrumental 


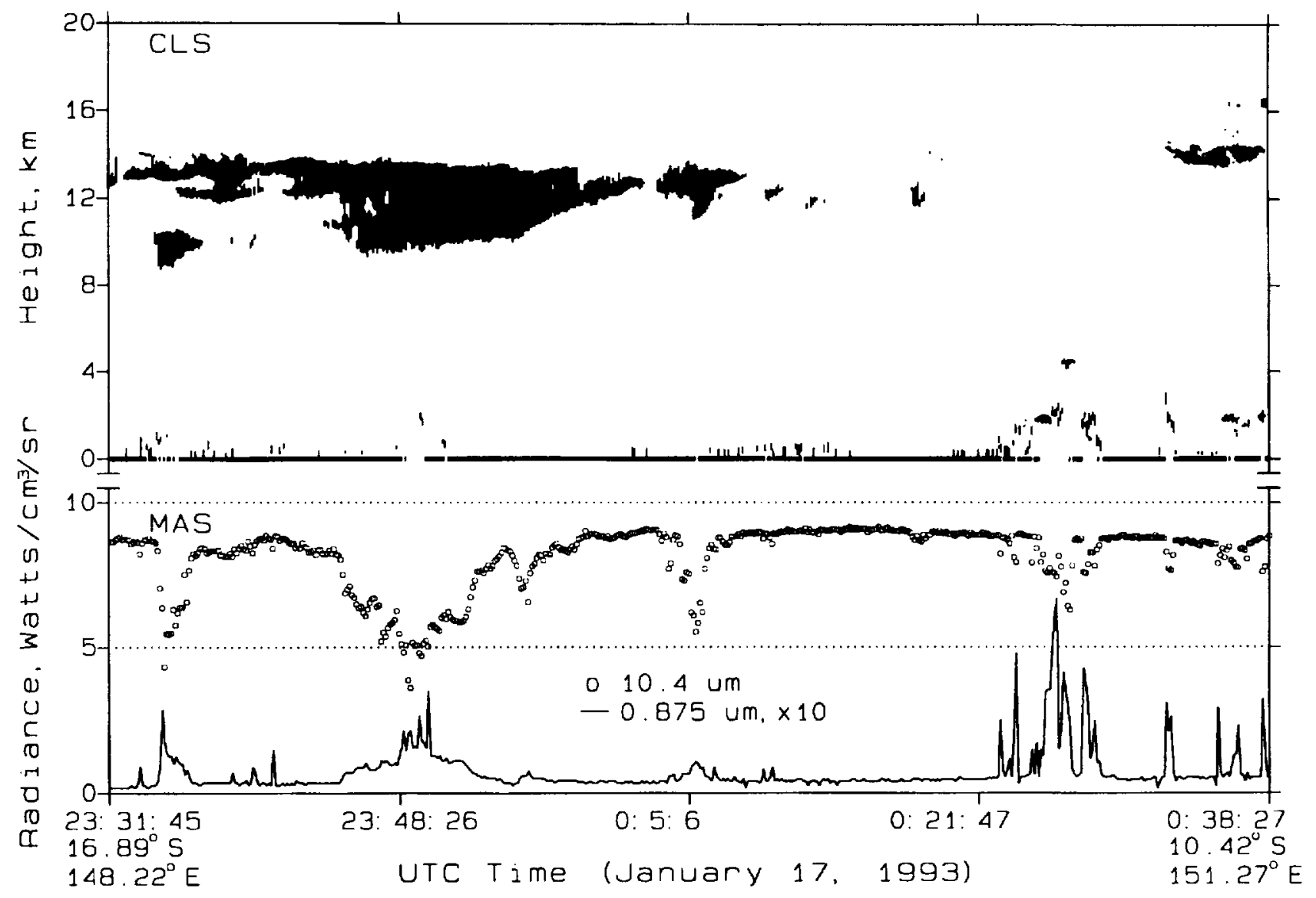

Fig. 1. Variations of reflectivity profiles from CLS and radiances from the 0.875 and $10.4 \mu \mathrm{m}$ channels of MAS.

problems, we deal with the differences in $T_{b}, \Delta T_{b}$, that directly result from the presence of the liquid clouds. Radiative transfer calculations are also made from both clear and cloudy conditions to estimate $\Delta T_{b}$ values in the presence of clouds. A number of CLW values distributed randomly between the altitude range of $0-5 \mathrm{~km}$ are assumed in the calculations. A polynomial regression is applied to the calculated $\Delta T_{b}$ 's and assumed CLW's to arrive at functional relations between these two parameters at each AMPR frequency. It is found that the response of the $10.7 \mathrm{GHz}$ channel to liquid clouds is small, both from observations and calculations. The response at $85 \mathrm{GHz}$, on the other hand, is found to depend strongly on the location of the liquid cloud layer and temperature profile. This dependence on location and temperature is also the reason that the algorithm for water vapor profiling could estimate CLW and the location of the liquid cloud layer from the MIR data. Therefore, only the calculated results at the 19.35 and $37 \mathrm{GHz}$ channels are used to compare with the AMPR observations to estimate CLW's. These CLW's are then compared with those estimated from the water vapor profiling with the MIR data in the next section.

\section{COMPARISON AND CONCLUSION}

Both CLW values and cloud tops estimated from the MIR water vapor profiling are compared with those estimated from AMPR and CLS measurements. A scatter plot between the CLS measured and MIR estimated cloud tops shows a lot of scatter of data points. This is mainly due to the limited capability of the millimeter-wave radiometric measurements to recover the fine structure of water vapor and liquid cloud distribution (millimeter-wave radiometric measurements are characterized by broad weighting functions). The cloud top determination is thus limited to a few selected levels during the water vapor profiling from the MIR data. When the CLS measured cloud tops corresponding to a given cloud top estimated from the MIR water vapor profiling are averaged, the comparison is found to be much improved.

The comparison of the CLW values estimated from MIR water vapor profiling and from the AMPR measurements also shows a lot of scatter of data points, although there is a definite correlation between the two estimated parameters. Aside from the MIR and AMPR measurement errors and uncertainty involved in the retrieval process, the lack of exact matching of 


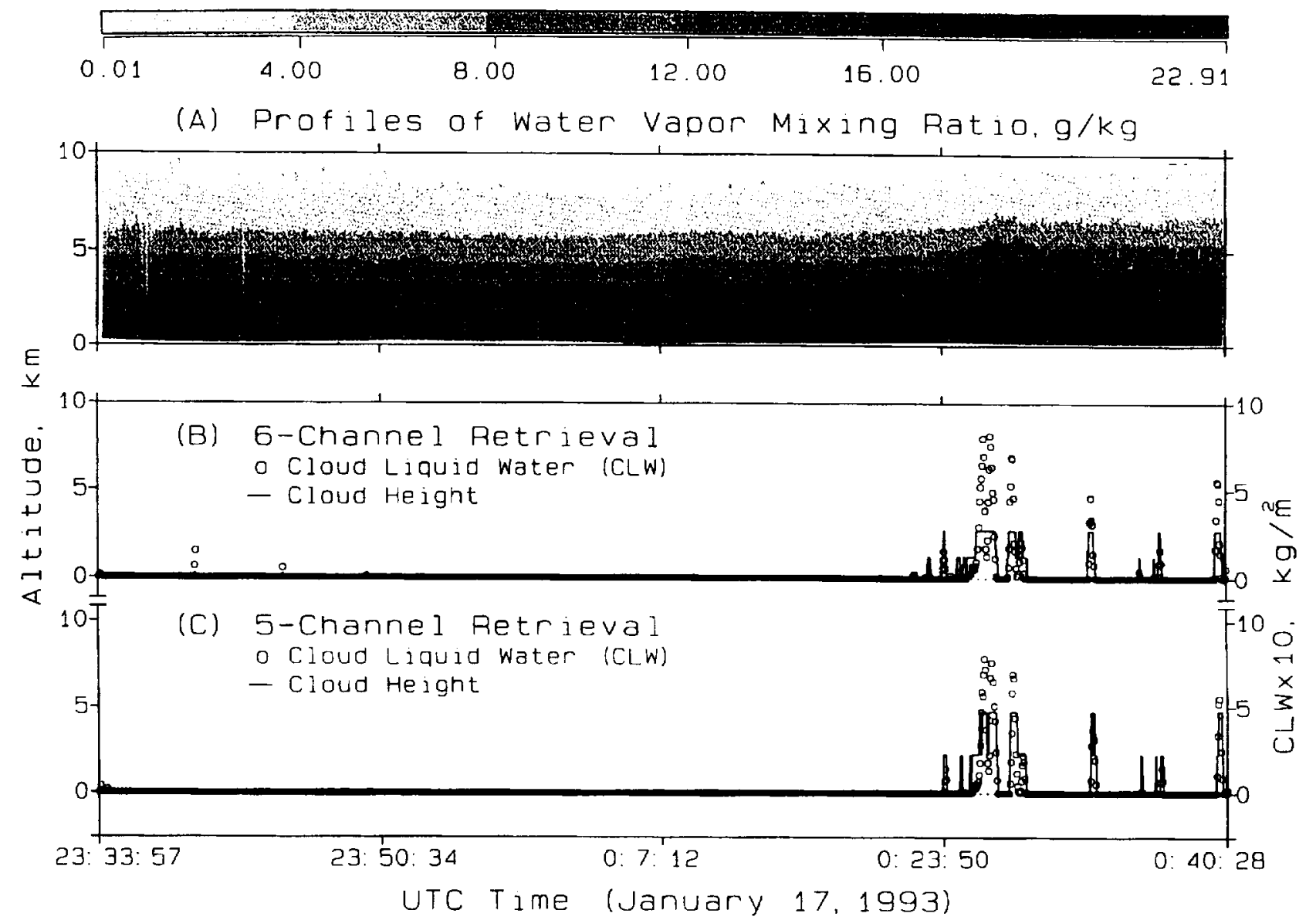

Fig. 2. The retrieved profiles of water vapor mixing ratio (A), and estimated CLW's and cloud tops using 6-channel (B) and 5-channels $(\mathrm{C})$ retrievals.

the observations between the two instruments could contribute to the scatter too.

It is concluded that the liquid cloud parameters estimated from the MIR water vapor profiling compare reasonably with those independently measured by the CLS and AMPR. This indirectly implies the robustness of the algorithm of water vapor profiling at the MIR frequencies. The scatter in the cloud top comparison is mainly caused by limitation in the passive millimeter-wave measurements.

\section{REFERENCES}

[1] J. R. Wang, J. D. Spinhirne, P. Racette, L. A. Chang, and W. Hart, "The effect of clouds on water vapor profiling from the millimeter-wave radiometric measurements," J. Appl. Meteor., 36(9), pp. 1232-1244, 1997.

[2] J. D. Spinhirne, W. D. Hart, and D. L. Hlavka, "Cirrus infrared parameters and shortwave reflectance relations from observations," J. Atmos. Sci., 53(10), pp. 1438$1458,1996$.
[3] M. D. King, W. P. Menzel, P. S. Grant, J. S. Myers, G. T. Arnold, S. E. Platnick, L. E. Gumley, S. C. Tsay, C. C. Moeller, M Fitzgerald, K. S. Brown, and F. G. Osterwisch, "Airborne scanning spectrometer for remote sensing of cloud, aerosol, water vapor and surface properties," J. Atmos. Ocean. Technol., 13(3), 777-794, 1996.

[4] J. R. Wang, S. H. Melfi, P. Racette, D. N. Whiteman, L. A. Chang, R. A. Ferrare, K. d. Evans, and F. J. Schmidlin, "Simultaneous measurements of atmospheric water vapor with MIR, Raman lidar, and rawinsondes," J. Appl. Meteor., 34, pp. 1595-1607, 1995.

[5] P. Racette, R. F. Adler, J. R. Wang, A. J. Gasiewski, D. M. Jackson, and D. S. Zacharias, "An airborne millimeterwave imaging radiometer for cloud, precipitation, and atmospheric water vapor studies," J. Atmos. Ocean. Technol., 13, 610-619, 1996.

[6] R. W. Spencer, R. E. Hood, F. J. LaFontaine, E. A. Smith, R. Platt, J. Gagliano, V. L. Griffin, and E. Lobl, "Highresolution imaging of rain systems with the advanced microwave precipitation radiometer," J. Atmos. Ocean. Technol., 11, 849-857, 1994. 